This item was submitted to Loughborough's Research Repository by the author.

Items in Figshare are protected by copyright, with all rights reserved, unless otherwise indicated.

\title{
Finite-element modelling of mechanical behaviour of rapid manufactured
} textiles

PLEASE CITE THE PUBLISHED VERSION

PUBLISHER

Professional Engineering Publishing / @ IMECHE

VERSION

VoR (Version of Record)

LICENCE

CC BY-NC-ND 4.0

\section{REPOSITORY RECORD}

Crookston, J.J., A.C. Long, Guy A. Bingham, and Richard J.M. Hague. 2019. "Finite-element Modelling of Mechanical Behaviour of Rapid Manufactured Textiles". figshare. https://hdl.handle.net/2134/4768. 
This item was submitted to Loughborough's Institutional Repository (https://dspace.lboro.ac.uk/) by the author and is made available under the following Creative Commons Licence conditions.

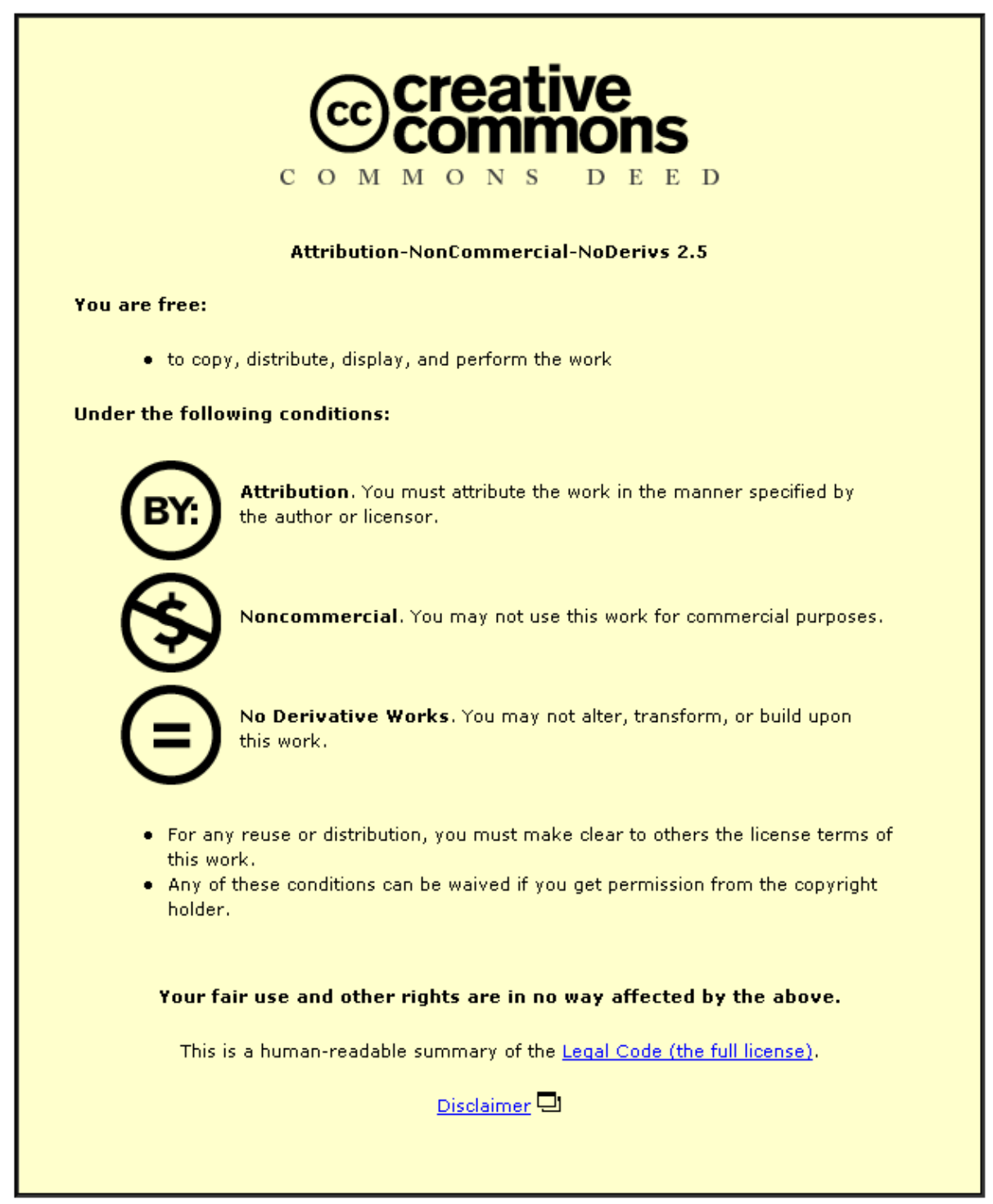

For the full text of this licence, please go to: http://creativecommons.org/licenses/by-nc-nd/2.5/ 


\title{
Finite-element modelling of mechanical behaviour of rapid manufactured textiles
}

\author{
J J Crookston ${ }^{1 *}$, A C Long', G A Bingham², and R J M Hague ${ }^{2}$ \\ ${ }^{1}$ Polymer Composites Research Group, School of Mechanical, Materials, and Manufacturing Engineering, University \\ of Nottingham, University Park, Nottingham, UK \\ ${ }^{2}$ Rapid Manufacturing Research Group, Wolfson School of Mechanical and Manufacturing Engineering, Loughborough \\ University, Leicestershire, UK
}

The manuscript was received on 17 May 2007 and was accepted after revision for publication on 4 September 2007.

DOI: 10.1243/14644207JMDA161

\begin{abstract}
Novel textile-like linked structures, realised using a rapid manufacturing (RM) process, have been proposed and developed recently. Various potential applications exist, and in most cases the mechanical performance must be evaluated. The present paper outlines a method to solve this problem by employing finite-element (FE) techniques at two scales, using the results of analysis at the meso-scale (the scale of the repeating unit) to provide equivalent non-linear spring behaviour for each textile link at the macro-scale. Validation with experimental test data from each scale was performed and these results are presented. Initial results overestimated mechanical performance. Microscopy suggested that this may be due to edge porosity in the specimens, and the dimensions of the FE models were adjusted accordingly as an initial approach to account for this. Predictions incorporating this modification exhibited excellent agreement with experimental measurements. The current work demonstrates both a technique, which could be automated for use in the design environment, and the potential value of developing an integrated process simulation to provide local material property data for the FE model.
\end{abstract}

Keywords: multiscale modelling, selective laser sintering, geometric mapping

\section{INTRODUCTION}

The development of layerwise additive manufacturing techniques has evolved from the age of rapid prototyping (RP) to one in which rapid manufacturing $(\mathrm{RM})$ is being used as a low-volume commercial production technique. The design freedom associated with additive processes enables the manufacture of discontinuous interlinked structures, which behave in a manner similar to textiles [1], as shown in Fig. 1. They can offer similar or even greater levels of out-of-plane and shear flexibility, while being manufactured from notionally homogeneous isotropic

*Corresponding author: Polymer Composites Research Group, School of Mechanical, Materials, and Manufacturing Engineering, University of Nottingham, University Park, Nottingham NG7 2RD, UK. email: jon.crookston@nottingham.ac.uk materials rather than highly anisotropic bundles of fibres. This provides some added benefits, which can be exploited, for example, when making protective clothing capable of absorbing local through-thickness impact loads due to the presence of hard surfaces joined together in a flexible manner (similar to the principles of medieval mail armour). The ability to incorporate other functionality such as fasteners (e.g. clips and zips) in a single manufacturing process also has great significance. Above all, the process lends itself to custom fitted items since there are no tooling costs associated with each design.

In collaboration with Loughborough University, a mapping technique building on the University of Nottingham's textile modelling expertise [2] was developed to enable arbitrary shapes to be covered with conformal RM textile structures in an automated fashion. This technique uses a surface mesh of the geometry similar to that used in finite element (FE) 


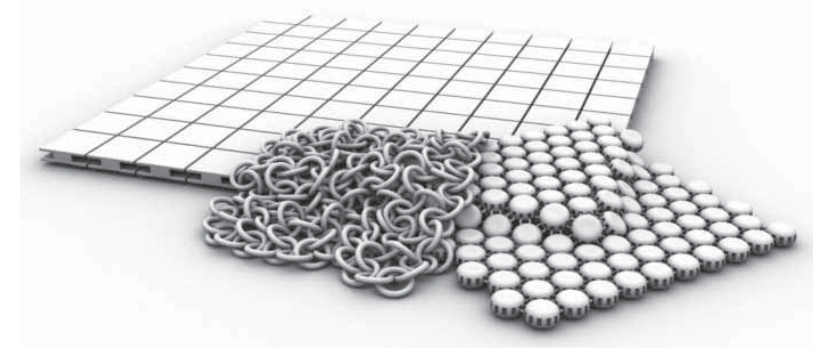

Fig. 1 Typical rapid manufactured textiles

analysis, and places the repeating link geometry at each of the nodes, such that interlinking loops lie along the element edges. This is shown schematically in Fig. 2. Provided that a sufficiently uniform mesh is used this is a robust technique. A mesh must be such that the textile geometry can be fitted to the nodes and element edges without significant deformation, i.e. the distributions of both element edge lengths and angles between element sides must be relatively smooth. The method is described in more detail in reference [3] and has been developed as an in-house software package known as ChainLink. Since the mapping process is now essentially automated, it is timely to determine an efficient route to evaluate mechanical behaviour of any proposed structures in order to complete the design process. This would enable both the prediction of structural performance and the optimization of textile repeating unit geometry. This is not a trivial problem due to the presence of rigid body movement in some loading conditions. Due to the novelty of the structures in question, appropriate modelling techniques have not yet been reported in the literature. Developing a suitable method for this problem, which may be applied in general situations is the focus of the present paper.

\section{MATERIAL BEHAVIOUR}

The material used for specimen manufacture was Duraform PA, a commercially produced semicrystalline nylon-12 powder, having an average particle diameter of approximately $58 \mu \mathrm{m}$. Studies have shown a significant dependence of mechanical (a)

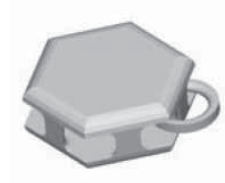

(b)

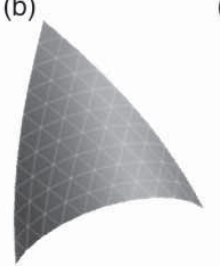

(c)

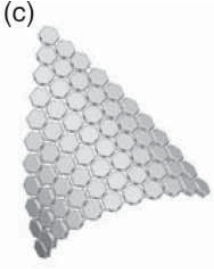

Fig. 2 (a) The basic unit of textile construction; (b) mesh of surface to be fitted with textile; and (c) conformal textile behaviour on temperature and ageing [4]; because of this, all mechanical testing was performed under the same atmospheric conditions $\left(T=20^{\circ} \mathrm{C}, \mathrm{RH}=\right.$ 50 per cent) and all mechanical test specimens were of the same age. Manufacturing was performed by selective laser sintering (SLS), a technique favoured since it produces net-shape parts, requiring no additional structure to support the layers as they are being built. The layerwise nature of the process has been shown to cause some inherent anisotropy [5]; this was not considered in the modelling work, but mechanical test specimens were all manufactured in the same orientation within the machine to avoid inconsistency. Dimensional constraints on part geometry for SLS are a function of process parameters including powder particle size and laser spot diameter. The smallest achievable feature size using the equipment in the current study was approximately $0.5 \mathrm{~mm}$, while the dimensional tolerance of the process was within $\pm 0.2 \mathrm{~mm}$.

In order to evaluate the behaviour of the finished material, tensile test specimens were manufactured using the SLS process, having dimensions in accordance with BS 2782. Testing was undertaken using an Instron 1195 universal testing machine. Sample extension was recorded using a clip-on extensometer, while data from the load cell were also logged using a PC interface. Nominal stress and strain were converted to true stress and logarithmic strain using the relations $[\mathbf{6}]$

$$
\varepsilon_{\text {true }}=\ln \left(1+\varepsilon_{\text {nom }}\right)
$$

and

$$
\sigma_{\text {true }}=\sigma_{\text {nom }}\left(1+\varepsilon_{\text {nom }}\right)
$$

where $\varepsilon$ is strain and $\sigma$ is stress; subscripts nom and true refer to nominal and true quantities, respectively. Three repeat specimens were tested; the resultant stress-strain data are shown in Fig. 3. The specimens showed elastic-plastic behaviour prior to a sudden failure exhibiting unstable crack growth and bifurcation. Localized necking was not observed prior to failure. An elastic-plastic material model was fitted to these stress-strain data, characterized by the material parameters in Table 1. Ultimate failure was described using a progressive damage model within the Abaqus FE code, specified using the Damage Initiation and Damage Evolution options. The damage model uses a non-local formulation implemented in terms of equivalent plastic displacement in order to eliminate mesh dependency. Further details of this technique can be found in the Abaqus documentation [6]. To verify the behaviour of the material model, a single three-dimensional FE (type C3D8) was subjected to a pure axial tensile 


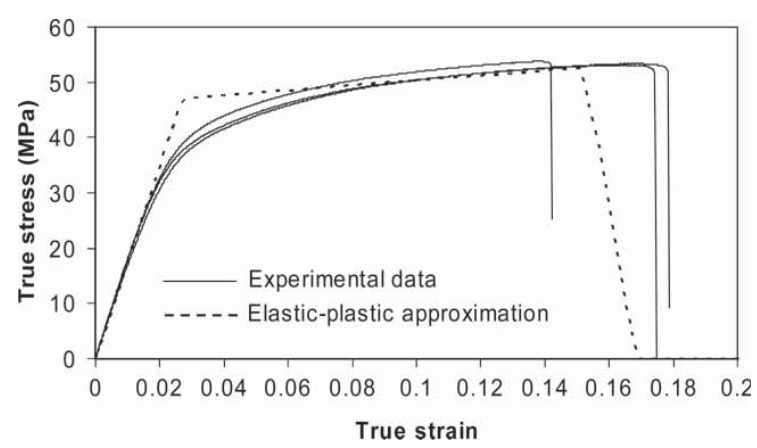

Fig. 3 Stress - strain data for Duraform PA obtained by tensile testing, with the assumed elastic-plastic model superimposed

stress, to represent the material within the gauge length of the tensile test specimen; the response is plotted with the experimental data in Fig. 3. Although the ultimate failure observed in the tensile specimens was caused by unstable crack growth, it was considered that using this ductile failure model with a suitably low damage evolution parameter provided a good description of the material behaviour. A damage evolution parameter of zero would result in fully brittle behaviour.

\section{APPROACH FOR MODELLING RM TEXTILES}

Since RM textiles consist of a large set of deformable, discrete, solid bodies with small feature sizes relative to the dimensions of the macroscopic assembly, it is not practical to simulate the deformation of a complete piece of material using a detailed model of solid FE. The large number of elements and contact surfaces required and the presence of rigid body motion make such a solution impossible with the computing hardware typically available to designers. Based on the strategy used for geometric mapping over complex surfaces, described above, an assembly of equivalent links can be considered, as indicated for a simple torus-based textile in Fig. 4. The method employed was to conduct an analysis of the meso-scale solid model in order to obtain the nonlinear force-displacement response; subsequently these data were used to define the non-linear spring behaviour of the equivalent link. Such a technique

Table 1 Parameters used in the elasticplastic material model

\begin{tabular}{lr}
\hline Young's modulus (MPa) & 1733 \\
Poisson's ratio [7] & 0.3 \\
Yield stress at 0 plastic strain (MPa) & 46.6 \\
Yield stress at 0.12 plastic strain (MPa) & 53.0 \\
Plastic strain at damage initiation & 0.1 \\
Damage evolution parameter & 0.05 \\
\hline
\end{tabular}

(a)

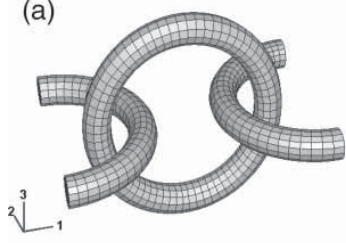

(b)

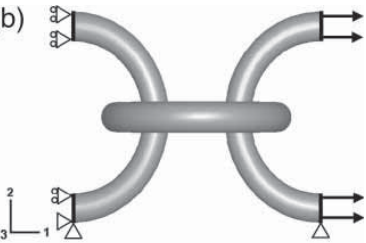

(c)

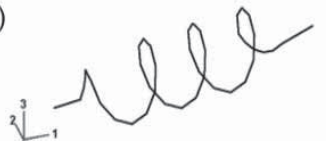

Fig. 4 (a) FE mesh of meso-scale link used to characterise the structural behaviour of a simple RM textile; (b) boundary conditions applied to the meso-scale link model (all faces having boundary conditions were also constrained in the third direction); and (c) abstraction to an equivalent single spring element

is appropriate for modelling the equivalent membrane behaviour of such textiles under tensile loads. Limitations exist for conditions where loading causes bodies to contact with one another in a friction dominated or unstable manner, i.e. compression or shear loads. For the latter case, the use of an additional spring element across the diagonal of a square of trusses has been suggested for conventional textiles used as composite reinforcements [8], but the use of such a technique for these structures would require some caution since the shear behaviour is dominated by different mechanisms from those affecting fibrous textiles.

Meso-scale FE analyses were conducted using the Abaqus Explicit code, primarily due to its ability to solve problems containing rigid body motions and to handle multiple contact conditions. The boundary conditions used are shown schematically in Fig. 4(b). Displacement boundary conditions were used in the interests of model stability. Automatic mass scaling was used to obtain a solution in a reasonable time without sacrificing accuracy; this is a standard technique when solving quasi-static problems using an explicit dynamic code. Standard checks were used to verify the validity of the analysis, including checking that the ratio of kinetic energy to internal strain energy in the model was small (always $<10$ per cent, typically approximately 2 per cent). A hard contact algorithm was used with no contact damping. Linear and quadratic global damping were applied to the model using the Bulk Viscosity option to reduce numerical artefacts in the solution. The linear and quadratic damping coefficients were 0.06 and 1.2, respectively. The model shown in Fig. 4(a) consisted of approximately 3300 nodes and 2500 hexahedral elements, and took approximately $15 \mathrm{~min}$ to run on a desktop PC with dual $2.8 \mathrm{GHz}$ Intel Xeon 
processors. The mesh density was selected on the basis of a simple convergence study for non-linear behaviour for this load case. For other loading conditions, which may introduce different localized stress concentrations, further investigation may be necessary.

The force-displacement history from the meso-scale model contained a certain amount of oscillation due to the dynamic nature of the solution technique, as can be seen in the results presented in Fig. 5. A polynomial function was fitted to the section of the curve between the occurrence of initial contact and final failure; in this initial study the order of the polynomial was selected manually to suit the characteristic shape of the curve without reproducing localized irregularities. This polynomial was used, in conjunction with the predicted failure displacement, to generate a table of force-displacement data in a suitable form for use with the Spring, Non-linear option within the Abaqus Standard implicit FE code. To verify the procedure, the output from a model consisting of a single Spring element is also plotted in Fig. 5.

Having determined the non-linear behaviour of the equivalent link, an analysis of a textile sample was conducted. The mesh of two-noded spring elements was generated by ChainLink from the same FE surface mesh used for construction of the solid model for manufacture. The models generated from ChainLink are illustrated in Fig. 6. The spring element mesh was written out in the Abaqus format and the non-linear spring behaviour and boundary conditions were added manually. All analyses incorporated large-displacement effects. Due to the simple nature of this test problem, the analysis was conducted on a single CPU in less than $1 \mathrm{~min}$. The results will be discussed in the following section of the current paper.

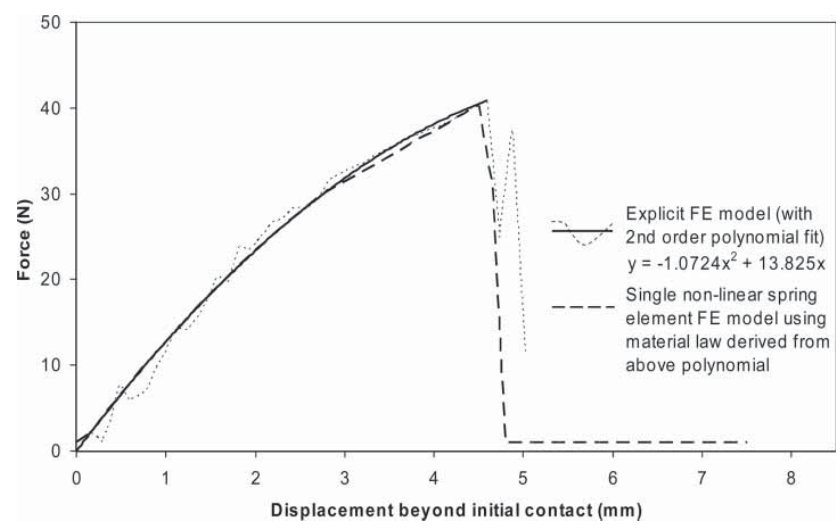

Fig. 5 Force-extension behaviour obtained from explicit FE analysis of the meso-scale link geometry. The coefficients of the polynomial fit were used to define the behaviour of a non-linear spring element; force-displacement data from a single element model are also shown (a)

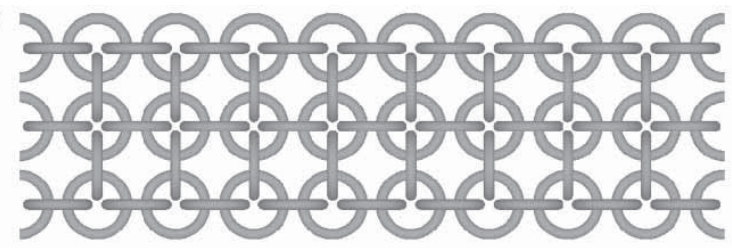

(b)

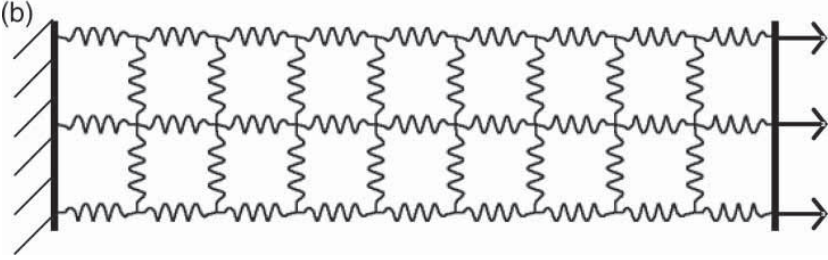

Fig. 6 (a) Three-dimensional geometry (without clamping blocks) for experimental testing and (b) abstraction of geometry using spring elements

\section{INITIAL RESULTS}

In order to verify the analysis results at each of the scales considered, comparisons were made with experimental measurements. Due to the dimensions of the meso-scale in typical RM textiles (e.g. $11 \mathrm{~mm}$ major diameter, $1.75 \mathrm{~mm}$ wire diameter for the torusbased textile illustrated in Fig. 6(a), validation of single link behaviour at this scale was performed using a larger realisation of a similar geometry, having a central link of $30 \mathrm{~mm}$ major diameter and $4.5 \mathrm{~mm}$ wire diameter. The specimens were manufactured with integrated clamping blocks suitable for the jaws of a testing machine. An as-manufactured specimen is shown in Fig. 7(a). To ensure that failure did not occur close to the clamping blocks, the tori connected to the blocks were manufactured with slightly larger dimensions than the central torus. Three repeat specimens were tested using the same equipment described above. Due to the constraint imposed by the presence of integrated clamping blocks, the boundary conditions applied to the model were modified to include restraint in the transverse direction, as illustrated schematically in Fig. 7(b). Since the deformation at the edge of the blocks was assumed to be negligible, the clamping blocks themselves were not modelled directly for reasons of computational efficiency.

Experimental results are presented in Fig. 8(a) and are compared with results from the FE model. It can be seen that the model significantly overestimated the stiffness and strength of the specimen; reasons for this are discussed in section 5 of this paper.

Using the method described in section 3, the non-linear spring behaviour of a meso-scale link was determined and the response of a piece of textile, shown schematically in Fig. 6, was predicted. The polynomial equation fitted to the meso-scale 
(a)

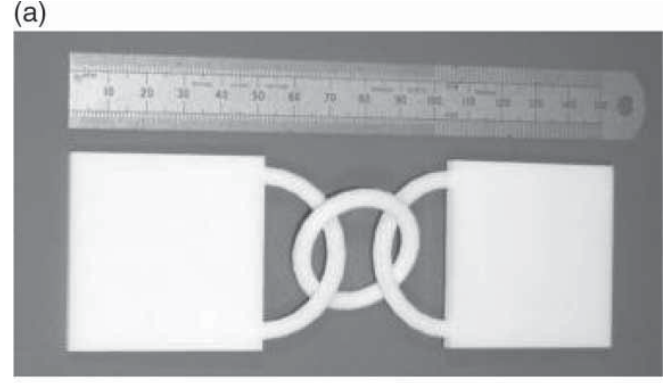

(b)

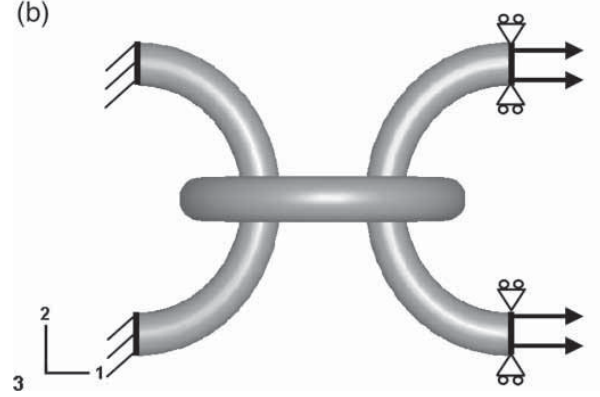

Fig. 7 (a) Large single link geometry used for validation at the meso-scale and (b) boundary conditions applied to the large meso-scale validation model. All faces having boundary conditions were fully constrained in the global 2 and 3 directions to simulate the effect of the clamping blocks

results was

$$
y=-1.0724 x^{2}+13.825 x
$$

where $x$ is the displacement in $\mathrm{mm}$ and $y$ is the force in Newton. The textile was manufactured with clamping blocks and tested in tension, once again using three repeat specimens. The experimental and predicted force-displacement responses of the textile are presented in Fig. 8(b). In both the textile and large single link specimens, failure was usually observed in the cross-sections of the links normal to the load, i.e. areas subjected to tension rather than bending; this was also the case in FE models. As in the case of the large single link geometry, the stiffness and strength were overestimated; reasons for this and ways to improve the agreement between model and experiment are investigated in the following section of the current paper.

\section{MESO-SCALE MODEL ADJUSTMENT AND JUSTIFICATION}

As stated above, initial predictions of component behaviour significantly overestimated stiffness and strength, although the material model showed good agreement with experimental data from tests on sintered tensile specimens. As mentioned previously,
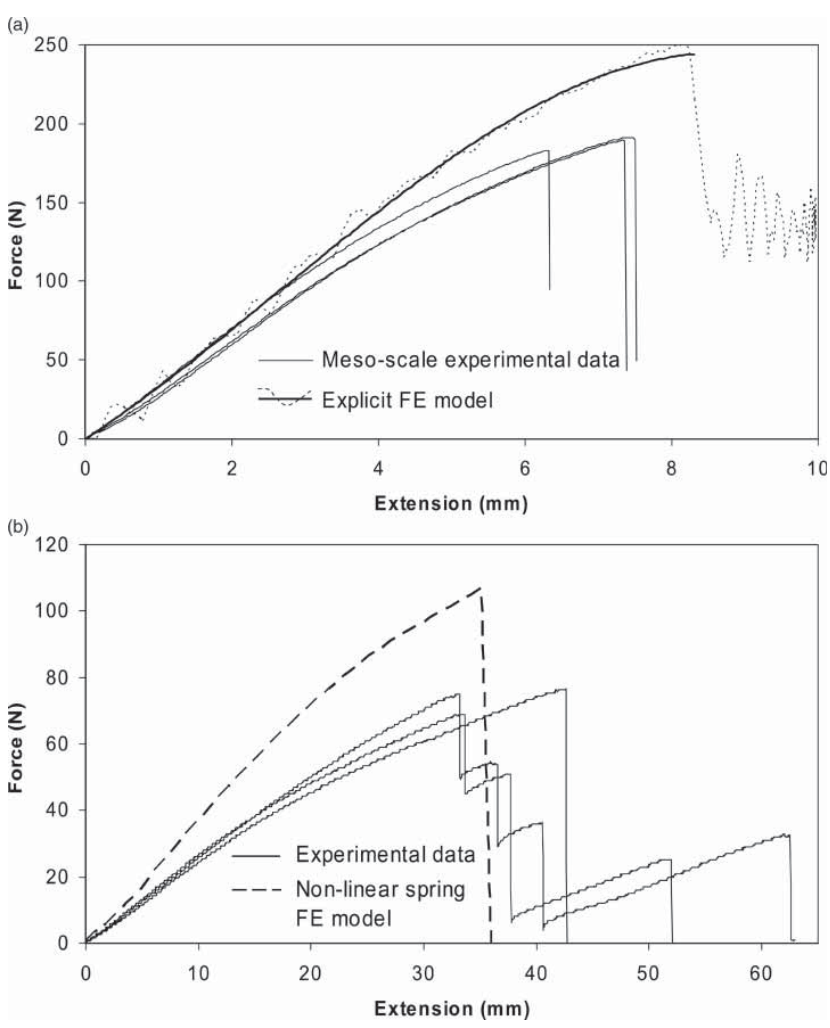

Fig. 8 Experimental force-extension response shown with FE predictions from the initial models for (a) the large single link geometry and (b) the textile specimen

published studies have noted that mechanical properties of this material can vary according to processing conditions and with orientation relative to the build direction [5]. Optical microscopy was performed on a central torus from an as-manufactured large single link geometry (as shown in Fig. 7). The torus was cast in a thermosetting polymer and ground and polished using standard sample preparation techniques, to permit examination of the material at the midplane. Care was taken to ensure that sufficient coolant was used during polishing to avoid overheating at the surface of the thermoplastic specimen. A dark dye penetrant was used to improve the contrast of voids against the white polymer. Microscopy was performed using a 5x objective lens; images were captured using a CCD camera connected to a PC.

It was observed that, although the majority of the specimen was well consolidated, some 'feathering' or edge porosity was observed, particularly in regions of the sample with edges at approximately $45^{\circ}$ to the build direction (i.e. the direction in which layers are added during manufacture). This phenomenon is illustrated in Fig. 9. Examination of material from the tensile specimens revealed very low porosity; this was probably due to the long, straight, and 

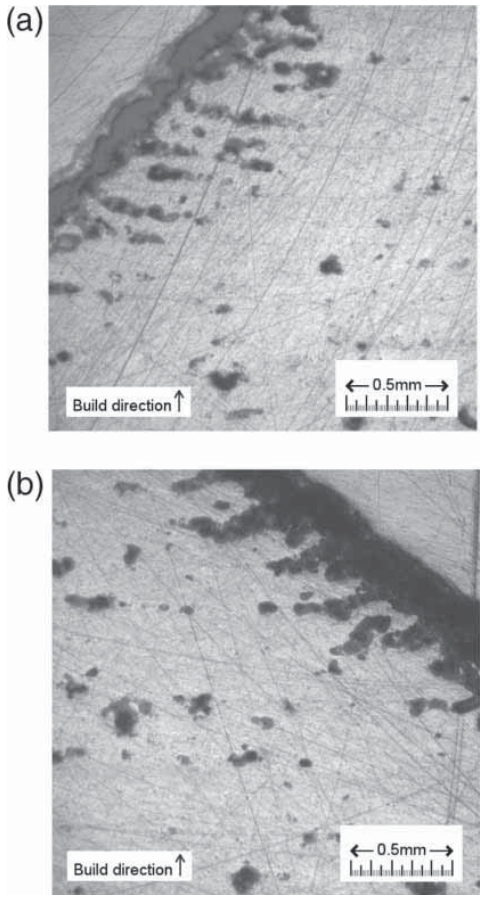

Fig. 9 Typical porosity and 'feathering' occurring at part edges

thick nature of the geometry, which resulted in greater heating and improved densification due to the higher volume to surface area ratio of the part.

This porosity could explain the difference in stiffness observed between experimental and predicted results. In a detailed study, it may be beneficial to employ a process model using techniques such as those described by Shi et al. [9], Bugeda et al. [10] and by Tontowi and Childs [11] and [12]; such a process model could estimate local porosity and provide appropriate local material properties for the FE analysis. Since this was beyond the scope of the present study a pragmatic approach was adopted, in which the wire diameters of the tori, which constituted the textile links were reduced by a notional value, effectively reducing the mechanical properties of the most porous regions to zero. In both the large and small single link models, the wire diameter was reduced by $0.5 \mathrm{~mm}$ as a first approximation based on the depth of the feathering observed at the link edges. Note that this value may differ for other geometries and process parameters, but appeared to hold for both torus-based geometries tested in the course of this work. While, in the presence of scaling effects in components of different dimensions, the use of a constant value to describe the reduction in effective size due to edge porosity will introduce changes in behaviour of different magnitudes, there are no inherent complications due to the general nature of the method.
The FE analyses were run with this new geometry. The revised polynomial equation fitted to the meso-scale results was

$$
y=-0.7801 x^{2}+9.6823 x
$$

where $x$ is the displacement in mm and $y$ is the force in Newton. Results from these revised models showed excellent agreement with experimental data, both for the large single link geometry and for the textile specimen. The predictions are presented in Fig. 10.

\section{DISCUSSION AND OUTLOOK}

It has been demonstrated that a two-scale modelling approach such as that described in this paper can be used to predict the response of these new structures. Since this is a new development in the field of RM applications, no previous analysis of such textiles has been performed. The method proposed can be applied to large RM textile structures due to the use of two separate modelling scales. Due to the nature of its operation much, if not all, of the analysis can be automated and driven by the application used to map the textile to the surface geometry. This will represent significant value as the suitability of such structures is assessed in the context of specific new applications.

However, porosity was observed around part edges, which must be accounted for in the model. The use of a simple reduction in wire diameter of the tori, which constituted these textiles was analogous to a surface erosion in the general case. Results using these models gave excellent agreement with experimental data, but this may not be the most robust method to account for the porosity since it does not give consideration to the actual geometry at the surface, particularly with respect to the build direction. After automating the technique, the next stage in development would be to incorporate a process model capable of predicting local geometry and porosity as a function of the processing parameters and hence able to provide material data at any given point within the model. This would enable optimization of the component according to requirements of structural performance, mass, and manufacturing time. A complicating factor with the textiles analysed herein is that the links are free to rotate after manufacture; hence, prediction of the effects caused by directionality resulting from the layered manufacturing process would probably be limited to the calculation of upper and lower bounds. It may be appropriate to apply a stochastic technique, for example accounting for build direction/orientation effects at the meso-scale to obtain a distribution of properties for use in a probabilistic model of the textile. It should be noted, however, that these 

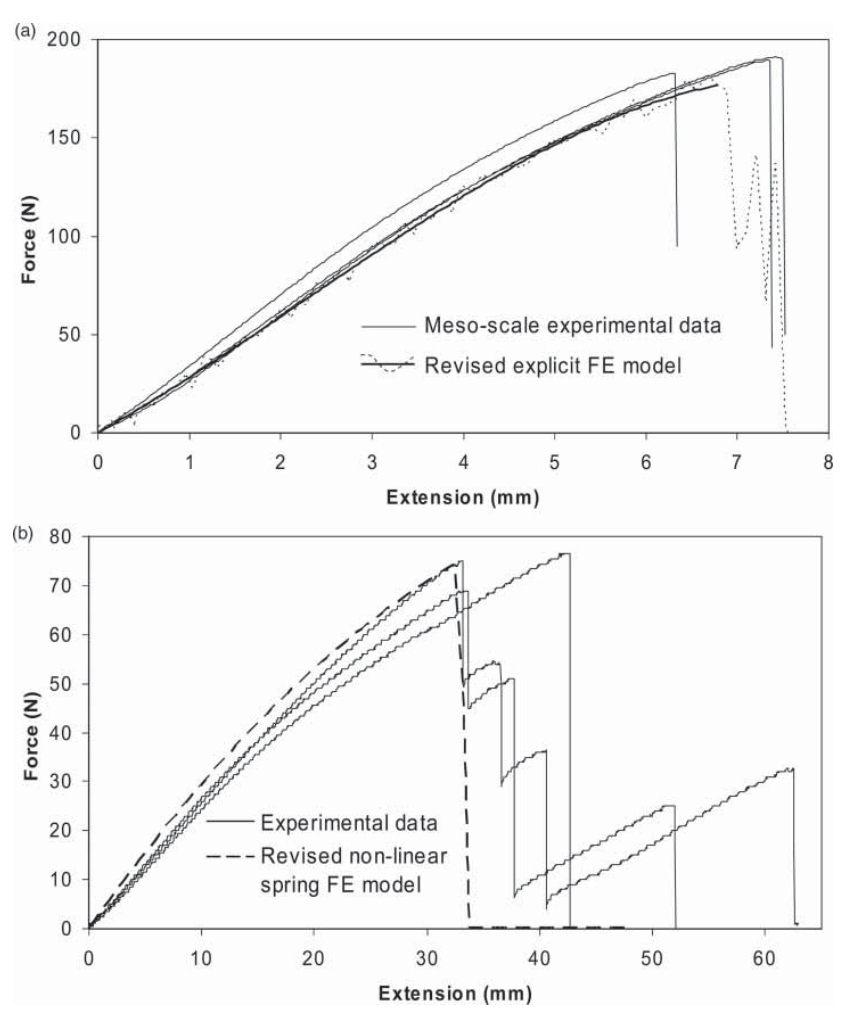

Fig. 10 Experimental force-extension response shown with $\mathrm{FE}$ predictions from the revised model having reduced wire diameters for (a) the large single link geometry and (b) the textile specimen

torus-based textiles were primarily employed as a simple model to demonstrate the technology, and few of the more complex textile forms exhibit the same freedom of rotation. Notwithstanding this statement a probabilistic method may also be effective, and more straightforward in implementation than a process model, at the meso-scale.

It should also be noted that the experimental data for some of the textile samples (Fig. 10(b)) exhibit a piecewise drop in force as failure occurs due to link breakages, which are not simultaneous across the width of the specimen. This behaviour is not present in the predicted results since all of the links were assigned identical mechanical properties. Contributions to the deviation from this idealized case in the experimental behaviour may be from variability in strength of the links due to the manufacturing process and from geometric misalignment in the specimens due to handling and friction at the contacts. Such imperfections could be introduced into the model at a later stage of development, particularly if a probabilistic method was employed to account for material variability.

It was noted that the use of one-dimensional spring elements would cause solver instabilities under certain loading conditions. However, some of these instabilities are present within the real material due to the friction dominated contact phenomena associated with compression and shear loads, in which the exact contact geometry is typically unknown. Since the structural performance of the material is exploited when subjected to tensile loads, it is anticipated that mechanical analysis, at least for design purposes, may be limited to such cases and that the appropriate in-service geometry (i.e. after any shear deformation or draping has occurred) should be analysed. Although through-thickness loads are relevant in applications such as protective clothing, such situations would require a different approach such as local stress analysis at the meso-scale; the present technique is intended for loading conditions where in-plane tension is dominant. Although some of the rigid body motions present may be modelled by defining the non-linear spring elements such that there is little resistance to movement within a particular range of extension, it may be more efficient to model some loading conditions using an alternative approach such as a kinematics or semi-rigid-body mechanics package.

The results presented in the current paper show that, at least in the context of an initial study, a nonlinear two-scale FE model has the potential to be used as a design tool for this new class of materials. Although further development of the methods would be required, initial validation is very promising.

\section{ACKNOWLEDGEMENTS}

The current work was undertaken at the University of Nottingham in collaboration with the University of Loughborough. Funding at both institutions was from their respective EPSRC Innovative Manufacturing Research Centres.

\section{REFERENCES}

1 Bingham, G. A., Hague, R. J. M., Tuck, C. J., Long, A. C., Crookston, J. J., and Sherburn, M. N. Rapid Manufactured textiles. Int. J. Comput. Integr. Manuf., 2007, 21(1), 96-105.

2 Sherburn, M., Long, A. C., Jones, I. A., and Rudd, C. D. TexGen: geometric modelling schema for textile composites. In Proceedings of the 8th International Conference on Textile Composites, Nottingham, UK, 16-18 October 2006, Paper T19.

3 Bingham, G. A., Hague, R. J. M., Crookston, J. J., and Long, A. C. Rapid manufactured textiles: a novel application for additive manufacturing. In Proceedings of 
the 17th Solid Freeform Fabrication Symposium (SFF 17), Austin, TX, USA, 14-16 August 2006.

4 Rapid Manufacturing Consortium Material Database. Available from www.rm-consortium.net, University of Loughborough, 2006.

5 Hague, R., Mansour, S., and Saleh, N. Material and design considerations for rapid manufacturing. Int. J. Prod. Res., 2004, 42(22), 4691-4708.

6 Abaqus, Inc., Abaqus 6.5 manual, 2004.

7 Faustini, M. C., Neptune, R. R., and Crawford, R. H. The quasi-static response of compliant prosthetic sockets for transtibial amputees using finite element methods. Med. Eng. Phys., 2006, 28(1), 114-121.

8 Yu, W. R., Zampaloni, M., Pourboghrat, F., Chung, K., and Kang, T. J. Sheet hydroforming of woven FRT composites: non-orthogonal constitutive equation considering shear stiffness and undulation of woven structure. Compos. Struct., 2003, 61(4), 353-362.
9 Shi, Y., Li, Z., Sun, H., Huang, S., and Zeng, F. Effect of the properties of the polymer materials on the quality of selective laser sintering parts. Proc. Instn Mech. Engrs, Part L: J. Materials: Design and Applications, 2004, 218(L3), 247-252.

10 Bugeda, G., Cervera, M., and Lombera, G. Numerical prediction of temperature and density distributions in selective laser sintering process. Rapid Prototyping J., 1999, 5(1), 21-26.

11 Tontowi, A. E. and Childs, T. H. C. Density prediction of crystalline polymer sintered parts at various powder bed temperatures. Rapid Prototyping J., 2001, 7(3), 180-184.

12 Childs, T. H. C. and Tontowi, A. E. Selective laser sintering of a crystalline and a glass-filled crystalline polymer: experiments and simulations. Proc. Instn Mech. Engrs, Part B: J. Engineering Manufacture, 2001, 215(B11), 1481-1495. 